\title{
Decaying Neutrinos in Galaxy Clusters
}

\author{
Adrian L. Melott and Randall J. Splinter \\ Department of Physics and Astronomy \\ and \\ Massimo Persic* and Paolo Salucci \\ SISSA and * Osservatorio Astronomic
}

\begin{abstract}
Davidsen et al. (1991) have argued that the failure to detect $u v$ photons from the dark matter DM) in cluster A665 excludes the decaying neutrino hypothesis. Sciama et al. (1993) argued that because of high central concentration the DM in that cluster must be baryonic. We study the DM profile in clusters of galaxies simulated using the Harrison-Zel'dovich spectrum of density fluctuations, and an amplitude previously derived from numerical simulations (Melott 1984b; Anninos et al. 1991) and in agreement with microwave background fluctuations (Smoot et al. 1992). We find that with this amplitude normalization cluster neutrino DM densities are comparable to observed cluster DM values. We conclude that given this normalization, the cluster DM should be at least largely composed of neutrinos. The constraint of Davidsen et al. can be somewhat weakened by the presence of baryonic DM; but it cannot be eliminated given our assumptions.
\end{abstract}

\section{Introduction}

Over a period spanning roughly the last ten years a number of papers have examined the astrophysical and cosmological consequences of a massive neutrino species possessing a specific radiative decay mode to photons with energies of roughly $15 \mathrm{eV}$ and at a rate of approximately $10^{-24} \mathrm{~s}^{-1}$. Melott (1984a) was able to provide an explanation for the morphological segregation of dwarf galaxies near large parent galaxies as seen by Einasto et al. (1974) by arguing that near the large parent galaxy the flux of $u v$ photons from neutrino decay would be larger and therefore one would expect that the satellite galaxies found nearer the parent galaxy should exhibit little neutral gas and hence little star formation. 
While those satellite galaxies further away from the parent galaxy would see a much smaller $u v$ flux and hence contain larger amounts of neutral gas. In addition Melott was able to derive the slope dividing the morphological types fitting, the observations of Einasto et al. (1974). In 1988 Melott et al. extended the model to provide explanations for another series of effects. Using a simple equilibrium argument they were able to show that with a neutrino of mass $\approx 30 \mathrm{eV}$ and lifetime $\approx 10^{24}$ seconds, that ionizing the universe to those levels required by the Gunn-Peterson test is completely reasonable in the neutrino decay scenario (see also Rephaeli \& Szalay 1981 and Sciama 1982). More recently Sciama (1990) has provided several additional problems that the neutrino decay model can provide a simple explanation for if $\tau \sim(1-3) \times 10^{23}$ sec.

Several attempts have been made for an unambiguous detection of the $u v$ flux from the neutrino decay, ever since the earlier theories of Cowsik (1977) and de Rujula \& Glashow (1980) (see Shipman \& Cowsik 1981; Henry \& Feldman 1981; Holberg \& Barber 1985; Fabian, Naylor \& Sciama 1991). In 1991 Davidsen et al., using the Hopkins Ultraviolet Telescope, performed a series of observations hoping to provide evidence for or against Sciama's (1990) decaying neutrino model. The Davidsen et al. experiment centered around the galaxy cluster A665. This cluster is known to be among the richest known clusters, therefore one would expect that it might possess a large dark matter (DM) halo. The DM halo consisting primarily of neutrinos would generate a $u v$ flux due to the decay of the neutrinos. The Davidsen experiment was unable to find any convincing evidence for the predicted $u v$ flux at the level anticipated from the decaying DM model, and they concluded that $\log \tau$ (seconds) $>24.5$ roughly. Furthermore they argued that the theory can only remain valid if one of the following conditions holds:(1) the cluster is several times less massive than estimated in their work (or there exists a significant baryonic dark matter component) and the redshifted decay photon energy happens to lie near the Ly $\beta$ airglow line, or (2) there is substantial absorption along the line of sight.

Sciama et al. (1993) argue that based upon several new pieces of evidence that assuming that all of the DM in the cluster is in the form of neutrinos may perhaps be an overly restrictive requirement. The first new piece of evidence concerns recent X-ray observations of A665. In 1992 Hughes \& Tanaka found strong evidence that the DM distribution in A665 is more centrally condensed than either the galaxy distribution or the hot X-ray emitting plasma. Sciama et al. (1993) argue for a baryonic form of DM in the central core of the cluster because the neutrinos being nearly collisionless would presumably be incapable of dissipating their energy enough to fall deeply into the potential of the cluster. Secondly, a calculation by Persic \& Salucci (1992) of the contribution of visual matter to $\Omega=\rho / \rho_{\text {crit }}$ shows that $\Omega_{\text {vis }} \approx 0.003$. Comparing this to the value of $\Omega_{\mathrm{B}} \approx 0.06 h_{50}^{-2}$ from primordial nucleosynthesis (e.g., Kolb \& Turner 1990; Peebles et al. 1991) argues for 
the existence of baryonic DM. With these two observations Sciama et al. concluded that it may be premature to argue that the Davidsen et al. observation conclusively rules out the decaying neutrino model.

Our goal in the present paper is to test the conjecture of Sciama et al. (1993) against a set of reasonable assumptions. We will do so by deriving detailed density profiles for the neutrinos using N-body experiments, which have initial density fluctuation amplitudes consistent with the recent COBE measurement (Smoot et al. 1992). Within this framework, this will enable us to make definite conclusions as to whether the neutrino DM is capable of providing the principal component to the DM distribution in the core of the cluster, and to assess the need for baryonic DM in the cluster core as required by Sciama et al. to evade the Davidsen et al. null result.

In this paper we use $H_{0}=50 h_{50} \mathrm{~km} \mathrm{~s}^{-1} \mathrm{Mpc}^{-1}$.

\section{Dark Matter in Cluster Cores}

Recent X-ray observations of clusters of galaxies imply that the DM distribution tends to be more centrally condensed than either the galaxy distribution or the distribution of the hot intra-cluster plasma (see, e.g., Sarazin 1992). In this section we wish to discuss the arguments for neutrino DM in the central cores of galaxy clusters, and in detail consider a recent model for the mass distribution in the cluster A665 which argues in favor of a baryonic component to the DM distribution in the core of the cluster A665.

Given the collisionless nature of the neutrinos it is often thought that the neutrinos are unable to fall deep into the cluster core. Doroshkevich et al. (1981) showed that when $k_{\mathrm{B}} T_{\nu}<<m_{\nu} c^{2}$ the characteristic velocity of the neutrinos is roughly $6.6\left(m_{\nu} / 30 \mathrm{eV}\right)^{-1}(1+z)$ $\mathrm{km} \mathrm{sec}{ }^{-1}$, which implies a characteristic temperature of $4.6 \times 10^{-5}\left(m_{\nu} / 30 \mathrm{eV}\right)(1+z) \mathrm{K}$ for a neutrino mass of $30 \mathrm{eV}$. This compared to typical dispersion velocities in clusters of order several thousand $\mathrm{km} \mathrm{sec}^{-1}$. Thus, the characteristic temperatures of the neutrinos is much smaller than the galaxies, and it appears that there may be no energetic problem with the neutrinos being trapped in the cluster potential well if the latter is varying with time in a suitable fashion (e.g., violent relaxation).

In 1979 Tremaine \& Gunn showed that one can place limits on the neutrino mass by phase space arguments. One might be tempted to believe that there might exist phase-space limits to the density of neutrinos in the cluster core, but if we use their limit on the neutrino mass 


$$
m_{\nu}>101 \mathrm{eV}\left(\frac{100 \mathrm{~km} / \mathrm{s}}{\sigma}\right)^{1 / 4}\left(\frac{1 \mathrm{kpc}}{r_{\mathrm{c}}}\right)^{1 / 4} g_{\nu}^{-1 / 4}
$$

where $\sigma$ is the the velocity dispersion and $r_{\mathrm{c}}$ is the core radius, and assuming typical quantities for a rich cluster (for instance $r_{\mathrm{c}} \approx 0.25 \mathrm{Mpc}$, and $\sigma \approx 10^{3} \mathrm{~km} / \mathrm{sec}$ ) we obtain a rather unrestrictive bound on the neutrino mass of $m_{\nu}>3.6 \mathrm{eV} g_{\nu}^{-1 / 4}$. Thus there do not exist any phase space limitations on neutrino DM in the core of a cluster.

Important advances have recently occurred in the understanding of the mass distribution in clusters of galaxies. In this section we will focus, in detail, on a recent mass model of A665.

Assuming hydrostatic equilibrium, X-ray data are in principle able to give the cluster's binding mass at any radius, $M(r)$. Given the gas density $\left(\rho_{\mathrm{g}}\right)$ and temperature $(T)$ profiles the expression

$$
M_{\mathrm{t}}(r)=\frac{k_{B} T r}{G \mu m_{p}}\left(\frac{d \log \rho_{g}}{d \log r}+\frac{d \log T}{d \log r}\right)
$$

gives $M(r)$ where all the quantities have their usual meaning (for a review see Sarazin 1986). While previous X-ray satellites lacked sufficient resolution to give $T(r)$, the latest generation of detectors (from, e.g., the Ginga and Rosat satellites) provide both $\rho_{\mathrm{g}}(r)$ and $T(r)$. Thus, one can separately deduce the mass in the hot intracluster plasma $M_{\mathrm{g}}(r)$, and the total (binding) mass, $M_{\mathrm{t}}(r)$. The distribution of mass locked in galaxies is usually estimated from the observed number counts distribution assuming an average galaxy mass (see for instance The \& White 1986). Recent observations of a number of clusters have yielded a striking and unexpected result: the DM distribution is characterized by a smaller core radius and a steeper slope than is the case for the gas and the galaxy distribution (Eyles et at. 1991; Briel et al. 1992; Gerbal et al. 1992; Hughes \& Tanaka 1992). This is exactly the opposite of what is observed in galaxies, where the DM is detected to be substantially more spatially extended than the visible matter (see Sciama et al. 1993), and could be interpreted as favouring dissipational baryonic DM in clusters.

For the cluster A665, we now discuss a self-consistent mass model derived from Hughes \& Tanaka (1992) and based on a combination of X-ray (Ginga) and optical data. The model includes:

1) A galaxy component of spatial density distribution, assuming $\left(M / L_{V}=20 h_{50}\right)$, 


$$
\frac{\rho_{\mathrm{G}}(r)}{\rho_{\text {crit }}}=\frac{750}{1+(r / 530 \mathrm{kpc})^{2}} .
$$

Note that Hughes and Tanaka assumed that the galaxies in the cluster have $M / L_{V}=5$. However, the extended halos around spirals and ellipticals certainly warrant a higher value. We assume a more realistic $M / L_{V}=20$ (e.g., Broeils 1992). This change increases the DM central density spike.

2) A hot-gas component,

$$
\frac{\rho_{\mathrm{g}}(r)}{\rho_{\text {crit }}}=\frac{1500}{1+(r / 380 \mathrm{kpc})^{2}},
$$

and the total (binding) mass distribution is (Hughes \& Tanaka 1992):

$$
\frac{\rho_{\mathrm{t}}(r)}{\rho_{\text {crit }}}=\frac{19700}{\left[1+(r / 298 \mathrm{kpc})^{2}\right]^{1.36}} .
$$

The DM density profile can be obtained from $\rho_{\mathrm{DM}}(r)=\rho_{\mathrm{t}}(r)-\rho_{\mathrm{g}}(r)-\rho_{\mathrm{G}}(r)$. The DM density profile is plotted in Figure 3, and from the figure it is clear that there is strong evidence for a substantial DM component in the cluster. Furthermore this DM component has a smaller core radius and is steeper than the corresponding density profile for the visible components. Equivalently, one might say that, compared to the visible mass components, the DM profile shows a central spike.

\section{Simulations}

The PM simulations (Hockney \& Eastwood 1980) were $128^{3}$ particles in $128^{3}$ cells with the Hot Dark Matter (hereafter HDM) initial power spectrum (e.g., Bond and Szalay 1983) with power spectrum $P(k) \propto k$ at small $k$. The amplitude of the initial perturbations is chosen to give the nonlinear autocorrelation $\xi(r) \propto r^{-1.8}$ at the moment we choose for our analysis so that it is in agreement with observational data on galaxy correlations (Peebles 1980). It is an extremely interesting coincidence that this normalization predicted the $(\Delta T / T)_{R M S} \approx 10^{-5}$ recently observed by COBE (Melott 1984b; Anninos et al. 1991). Predictions of higher amplitudes for the microwave background fluctuations in HDM models have been based on linear theory, not full nonlinear N-body simulations. 
There are, of course, well-known problems with the HDM model. Galaxies are expected to form only in the filamentary or sheetlike "pancakes" that form (Fig. 1), because compression takes place only in these sites. When this restriction is made, the autocorrelation amplitude of the material that remains is too high to be compatible with that of galaxies (White et al. 1983). Therefore we must appeal to some other process than gravitational instability in the pancake theory to make galaxies, for example some sort of distribution of seeds. Hopes that radiation pressure from the decaying neutrinos might start the (Hogan 1992) process have not worked, basically because the $u v$ ionizes the universe more efficiently than it heats it (Splinter and Melott 1992). Perhaps a model in which galaxies are seeded by independent fluctuations is necessary (Scherrer et al. 1989; Villumsen et al. 1991). At any rate, we take the COBE amplitude, which produces a mass autocorrelation slope and amplitude compatible with the present galaxy correlation amplitude, and beg the question of galaxy formation. The result will be a lower bound to the DM density in clusters, since additional power introduced on small scales would intensify the clustering.

In order to clearly understand any impact of boundary conditions (finite box size) or dynamical resolution, we simulated three cubical volumes, one $32 \mathrm{Mpc} h^{-2}$, one $64 \mathrm{Mpc}$ $h^{-2}$ and one $128 \mathrm{Mpc} h^{-2}$ on a side. Any interaction with computational volume effects should betray itself by giving a systematical difference in cluster properties.

Since this model is not hierarchical, but has a truncated initial power spectrum, there are discontinuities in the description of objects corresponding to voids, sheets, filaments, and clusters. We were able to identify clusters by looking for density maxima $\rho / \rho_{\mathrm{c}}>100$ and examining their properties. Since caustics are not resolved, they do not complicate the process.

After locating the highest density region of each cluster, we binned density into spherical shells surrounding that peak to generate a density profile. The properties of such simulated clusters will be compared in the next section with those of actual clusters (and notably A665).

We found impressive agreement over nearly the entire range of radii, except that smaller boxes with their higher resolution gave a different result at small $r$ outside the dynamic range of the larger box. We found all three curves agreed remarkably well down to a radius of about 0.3 cells, considerably better than we expected. We therefore show in Fig. 2 the averaged density profile, for the small box down to $r=6.25 \times 10^{-2} \mathrm{Mpc} h^{-2}$, the smallest reliable value. The errors are one standard deviation from one cluster to another in the mass density in a shell. 
In agreement with the argument suggested in section II on neutrinos being trapped in clusters, we see that the neutrinos are able to cluster into clumps of rather high central density with radially extended density profiles. An analytic fit to the average density profile of the simulated clusters yields:

$$
\frac{\rho_{\mathrm{DM}}(r)}{\rho_{\text {crit }}}=\frac{2200 \pm 700}{\left[1+\left(r / 910 h_{50}^{-2} \mathrm{kpc}\right)^{2}\right]^{1.17}}
$$

where the uncertainty represents the estimated range of central densities for our set of different cluster realization (see Fig.3).

In Fig. 3 we plot the individual neutrino density distribution of HDM-simulated clusters (solid lines) vs the "observed" DM density distribution of A665 (open squares). This predicted density profile appears to agree well with the distribution of mass in A665 at small radii. At large radii there are large discrepencies, but note at these large radii the observations have large errors. This is despite the fact that we assumed an initial amplitude for the density fluctuations that is consistent with the COBE observation. If, as suggested recently, some of the observed COBE amplitude might in part be due to gravitational waves (see, e.g.: Davis et al. 1992; Liddle \& Lyth 1992; Lidsey \& Coles 1992; Lucchin et al. 1992; Salopek 1992; Souradeep \& Sahni 1992), then the amplitude of our density profiles could be lowered, making them somewhat more compatible with observations. In any case the possibility of two-components to the DM distribution remains open: a baryonic component with an overdensity greater than $10^{4}$ dominating the innermost central $300 \mathrm{kpc}$, plus an exotic component (e.g., the neutrinos described by eq.[1]) dominating elsewere, fits DM density distribution in A665 quite well, but note a large baryonic contribution to the DM distribution is not necessarily required as demonstrated by our simulations. Therefore the neutrino interpretation of the cluster DM is still viable in the light of recent observational progresses and it is pertinent to investigate the limits on the timescale decay implied by the Davidsen et al. (1991) null result.

\section{Decaying Neutrinos}

The neutrinos contributing to the flux observed by HUT are contained in the volume defined by sliding the HUT window along the line of sight from one edge of the cluster to the other. The actual expression of the density distribution is required for an accurate estimate of the total flux: since two dimensions of the volume considered are quite small 
(and in any case comparable with the core radius of the DM distribution), the value of the central density is important; and since the third dimension extends to the cluster edge, the radial profile of the DM plays an important role as well. In detail, in order to compute the DM mass comprised within the cluster volume accessible to HUT, we have integrated the DM distributions, corresponding respectively to our A665 model and to our simulation, over the volume defined by the rectangular HUT window $\left(A_{\mathrm{HUT}}=68 \times 457 \mathrm{kpc}^{2}\right.$ at the distance of A665) and by a radial edge of $3 \mathrm{Mpc}$.

Assuming first that all the DM resulting from our mass decomposition is made by decaying neutrinos, we obtain a mass similar to that estimated by Davidsen et al. (1991). Since the flux is proportional to the mass (see below), from our mass model we predict a flux which is also similar to (slightly lower than) that estimated by Davidsen et al. (1991), which similarly implies a lower limit on the decay time of

$$
\tau_{23}>(6-20)\left(\frac{29 \mathrm{eV}}{m_{\nu}}\right)
$$

(with $\tau_{23}$ the neutrino lifetime in units of $10^{23}$ secs), also in accord with Davidsen et al. (1991).

On the other hand, according to our simulations the neutrino density in clusters follow the mean relationship given by eq.(1). This implies, by the same procedure as used above, a total mass in neutrinos (integrated over the HUT slit) about 2 times lower than the Davidsen et al. value: $(1.3 \pm 0.4) \times 10^{13} M_{\odot}$. So the predicted neutrino flux from the simulated cluster taken to be representative of A665 will be correspondingly lower than Davidsen et al.'s expected value:

$$
F_{\nu}=(0.039 \pm 0.013)\left(\frac{A}{0.068 \mathrm{kpc} \times 0.457 \mathrm{kpc}}\right)\left(\frac{f}{1.5}\right)\left(\frac{\rho_{\mathrm{o}}}{2000 \rho_{\mathrm{c}}}\right)\left(\frac{R_{\text {core }}}{0.3}\right)\left(\frac{\epsilon}{14 \mathrm{eV}}\right)^{-1} \tau_{23}^{-1}
$$

where $A$ is the area of the detector slit $f \simeq 1.5$ accounts for the radial variation of the density inside the HUT window, $\rho_{0}$ is the central density, $R_{\text {core }}$ is the DM core radius in $\mathrm{Mpc}, \epsilon$ is the monochromatic energy of the decay photons, $\tau_{23}$ is the neutrino lifetime in units of $10^{23} \mathrm{~s}$, and $F_{\nu}$ is in units of photons $\mathrm{s}^{-1} \mathrm{~cm}^{-2}$. Scaling the Davidsen et al. value of $\tau_{23}$ to the mass (or flux) of our simulated cluster we then get a tighter lower limit for the decay timescale:

$$
\tau_{23}>(3 \pm 1)\left(\frac{29 \mathrm{eV}}{m_{\nu}}\right)
$$


This limit is a factor 2 smaller than the one originally obtained by Davidsen et al. (1991), in that while they assume that all the central DM is neutrino for the purpose of computing the flux, we take the alternative view of estimating the minimal flux that in our scenario neutrino must emit from this cluster, independently of whether some baryonic DM resides in their innermost regions. We emphasize at this point the possibility that baryonic DM, although quite a minor component by mass fraction cluster-wide, might still dominate the inner $\sim 100 \mathrm{kpc}$ and show up as a central density spike. Our constraint, i.e. strictly $\tau_{23}>2\left(m_{\nu} / 29 \mathrm{eV}\right)^{-1}$, can be compatible with part of Sciama's (1990) range of values for $\tau_{23}$, and of course easily the original (Melott 1984a) suggestion of $10^{24} \mathrm{~s}$.

Computations were performed at the National Center for Supercomputing Applications, Urbana, Illinois. ALM gratefully acknowledges the support of NSF grants AST-9021414 and NSF EPSCoR Grant number OSR-9255223 and NASA grant NAGW-2923. RJS would like to thank NASA for providing support through a NASA Graduate Student Fellowship.

\section{REFERENCES}

Anninos, P., Matzner, R., Tuluie, R. \& Centrella, J. 1991, ApJ, 382, 71.

Bond, J.R., \& Szalay, A.S. 1983, ApJ, 274, 443.

Briel, U.G., Henry, J.P., \& Bohringer, H. 1992, A\&A, 259, L31.

Cowsik, R. 1977, Phys Rev Lett, 39, 784.

Davidsen, A.F., Kriss, G.A., Ferguson, H.C., Blair, W.P., Bowers, C.W., Dixon, W.V., Durrance, S.T., Feldman, P.D., Henry, R.C., Kimble, R.A., Kruk, J.W., Long, K.S., Moos, H.W., \& Vancura, O. 1991, Nature, 351, 128.

Davis, R.L., Hodges, H.M., Smoot, G.F., Steinhardt, P.J., \& Turner, M.S. 1992, Phys. Rev. Lett., 69, 1856.

de Rujula, A., \& Glashow, S.L. 1980, Phys Rev Lett, 45, 942.

Doroshkevich, A.G., Zel'dovich, Ya.B., Syunyaev, R.A., \& Khlopov, M.Yu. 1981, Sov. Astron. Lett., 6, 257.

Einasto, J., Saar, E., Kaasik, A., \& Chernin, A.D. 1974, Nature, 252, 111.

Eyles, D., et al. 1991, ApJ, 376, 23.

Gerbal, D. Durrent, F., Lima-Neto, G., \& Lachièze-Rey, M. 1992, A\&A, 253, 77.

Henry, R.C., \& Feldman, P.D. 1981, Phys Rev Lett, 47, 618. 
Hockney, R.W., \& Eastwood, J. 1981 Computer Simulation Using Particles (New York: McGraw-Hill).

Hogan, C.J. 1992, Nature, 359, 40.

Holberg, J.B., \& Barber, H.B. 1985, ApJ, 292, 16.

Klypin, A.A., \& Shandarin, S.F. 1983, MNRAS, 204, 891.

Kolb, E.W., \& Turner, M.S. 1990, in The Early Universe (Addison-Wesley Publishing Company).

Liddle, A.R., \& Lyth, D.H. 1992, Phys. Lett., B291, 391.

Lidsey, J.E., \& Coles, P. 1992, MNRAS, 258, 57P.

Lucchin, F., Matarrese, S., \& Mollerach, S. 1992, ApJ, 401, L49.

Melott, A.L. 1984a, Sov Astron, 28, 478.

Melott, A.L. 1984b, Sov Astron, 28, 631.

Melott, A.L., McKay, D.W., \& Ralston, J.P. 1988, ApJ Lett, 324, L43.

Peebles, P.J.E. 1980 The Large-Scale Structure of the Universe (Princeton: Princeton University Press).

Peebles, P.J.E., Schramm, D.N., Turner, E.L., \& Kron, R.G. 1991, Nature, 352, 769.

Persic, M., \& Salucci, P. 1992, MNRAS, 258, 14P.

Rephaeli, Y., \& Szalay, A.S. 1981, Phys. Letters, 106B, 73

Salopek, D.S. 1992, Phys. Rev. Lett., 69, 3602.

Sarazin, C.L. 1992, Clusters and Superclusters of Galaxies, ed. A.C. Fabian (Dordrecht: Kluwer Academic Publishers).

Scherrer, R.J., Melott, A.L., \& Bertschinger, E. 1989, Phys Rev Lett, 62, 379.

Sciama, D.W. 1982, MNRAS, 198, 1P.

Sciama, D.W., Persic, M. \& Salucci, P. 1993, PASP, 105, 102.

Sciama, D.W. 1990, ApJ, 364, 549.

Shipman, H.L., \& Cowsik, R. 1981, ApJ, 247, L111.

Smoot, G.F. et al. 1992, ApJ Lett, 396, L1.

Souradeep, T., \& Sahni, V. 1992, Mod. Phys. Lett., A7, 3541.

Splinter, R. \& Melott, A.L. 1992, ApJ, 394, 7.

Tremaine, S., \& Gunn, J.E. 1979, Phys. Rev. Lett., 42, 407. 
Villumsen, J.V., Scherrer, R.J., \& Bertschinger, E. 1991 ApJ, 367, 37; and erratum in 1991 ApJ, 381, 601.

White, S.D.M., Frenk, C.S., \& Davis, M. 1983 ApJ Lett, 274, L1.

This preprint was prepared with the AAS LATEX macros v3.0.

Fig. 1.- A plot of the particles in a thin slice of our medium simulation of a neutrinodominated universe.

Fig. 2.- The average density profile of DM in clusters in our high resolution box (box size $32 \mathrm{Mpc}$ ). The horizontal lines indicate one standard deviation in the density.

Fig. 3.- The DM density distribution of A665 (open squares) and the neutrino density distribution from our high resolution simulation (solid lines). Observations are uncertain for $R>2 \mathrm{Mpc}$, and the simulation can not resolve the innermost $\simeq 500 \mathrm{kpc}$. 


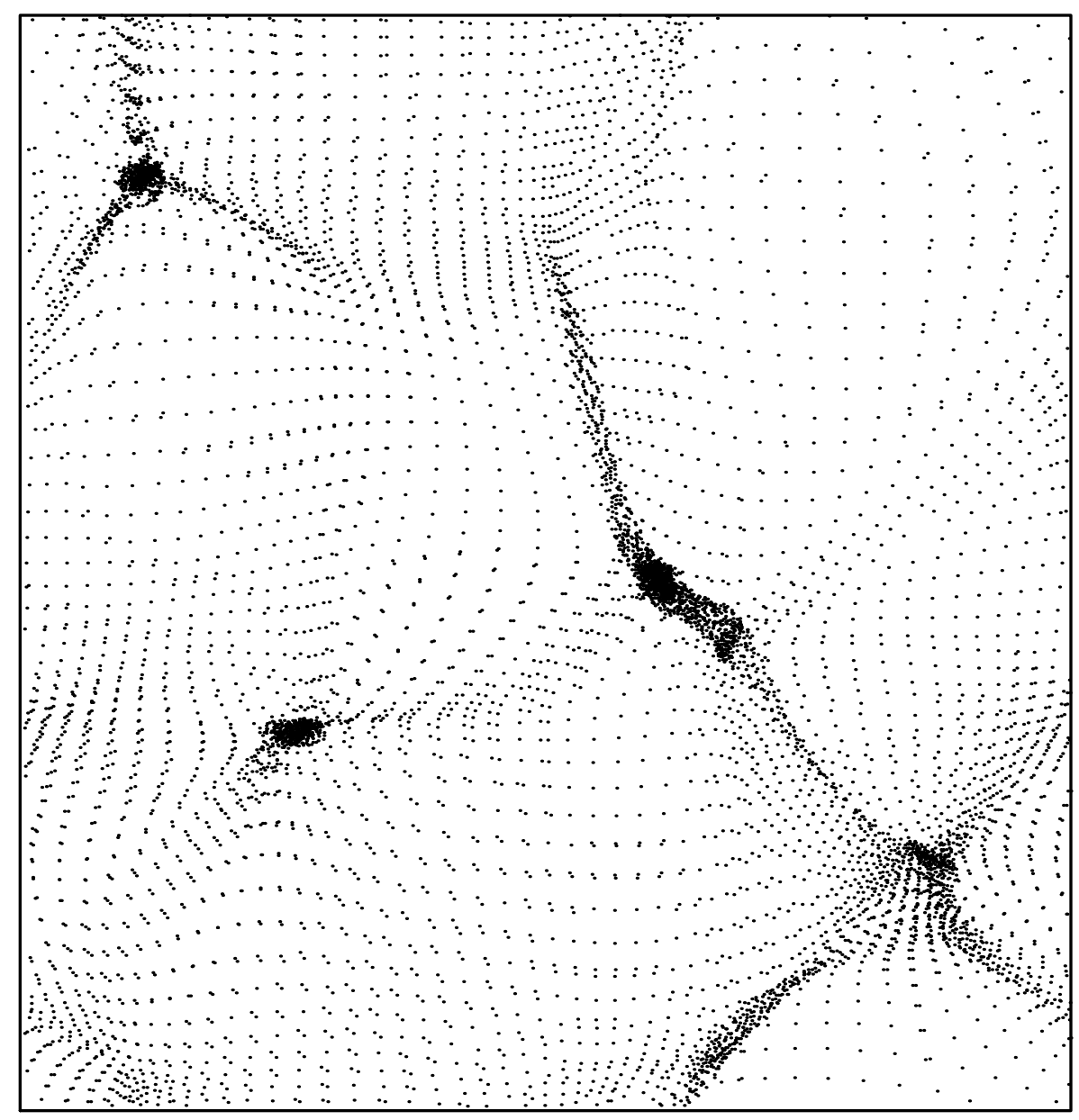




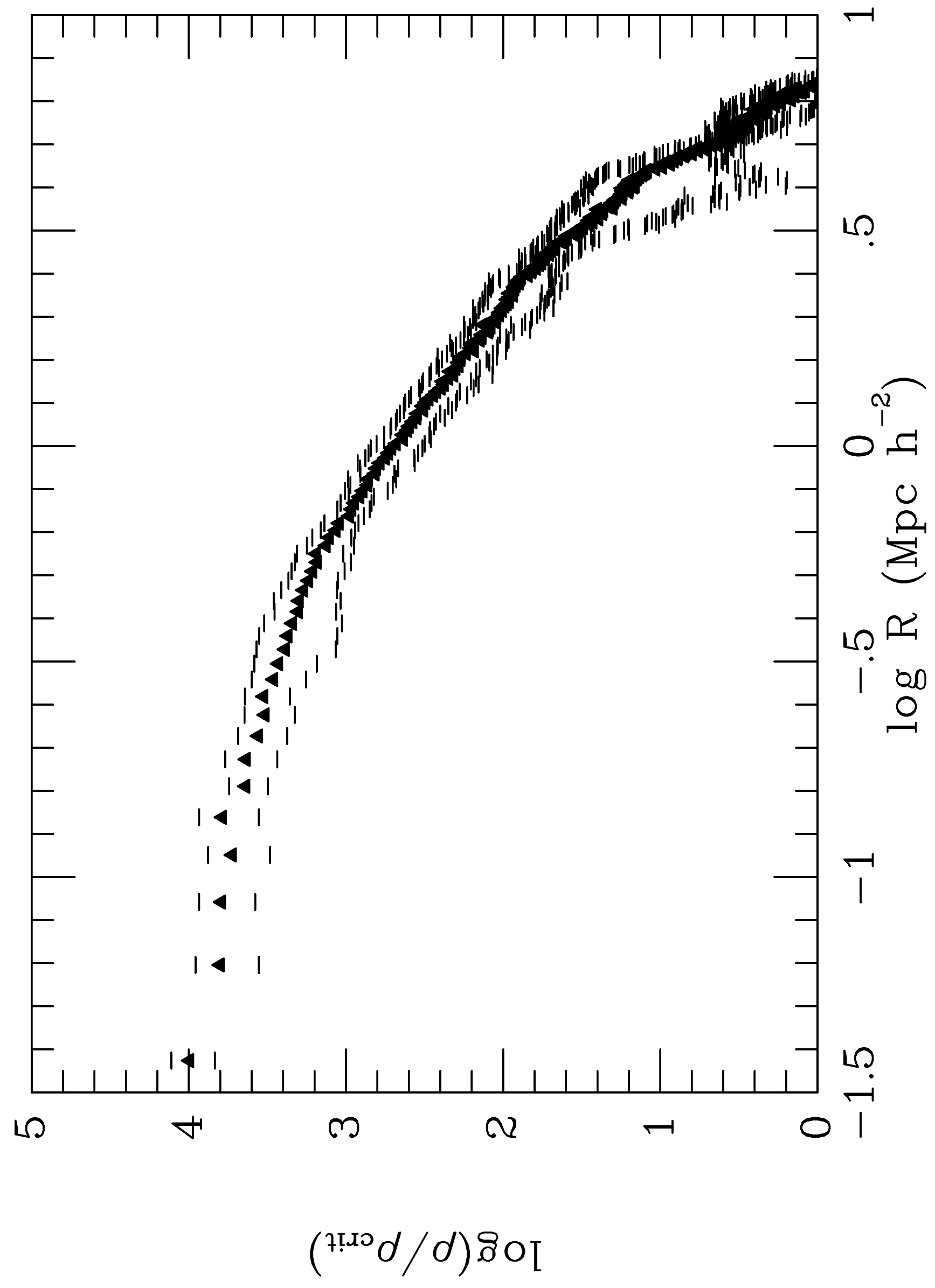




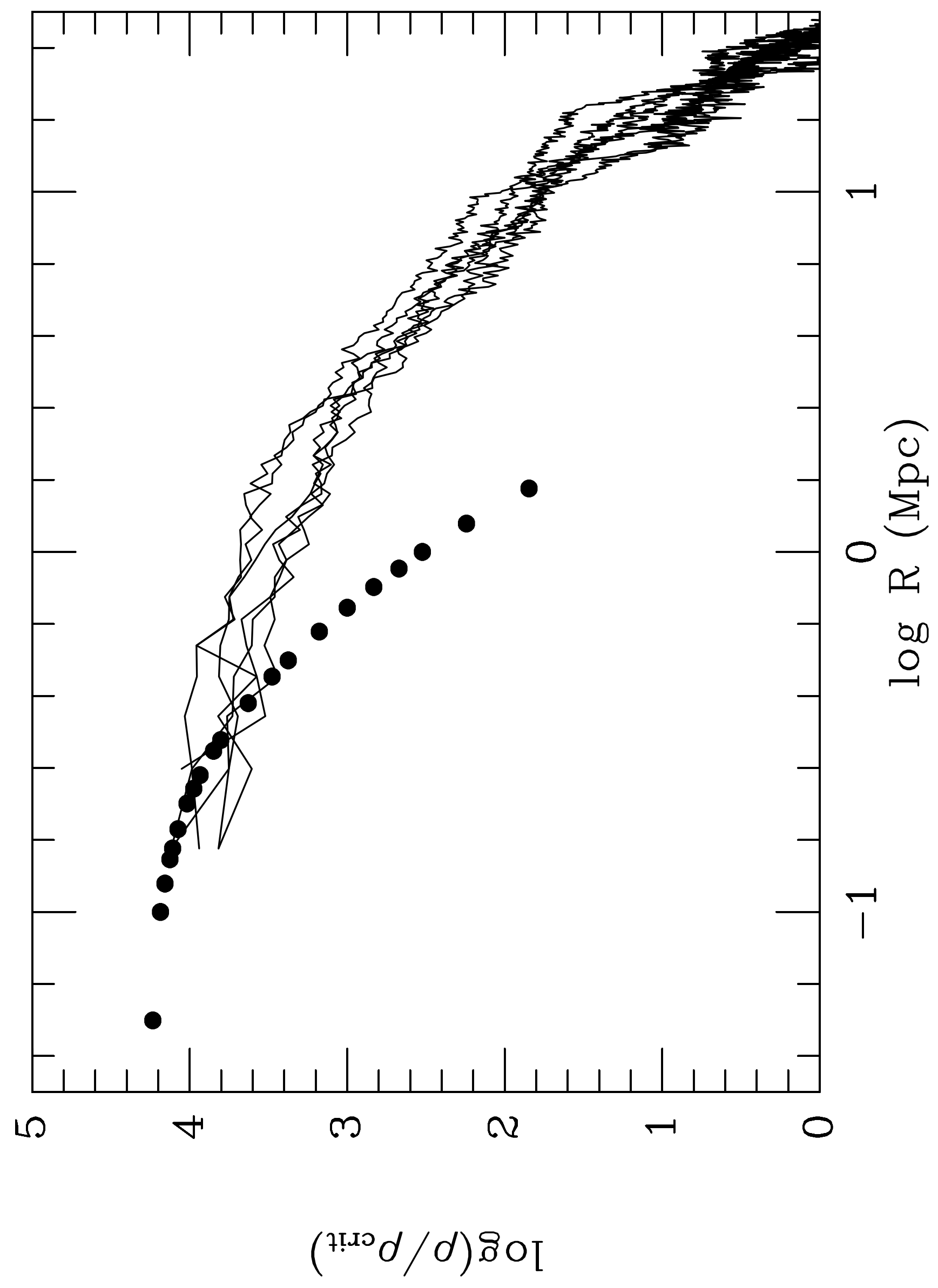

